\title{
Fusion of morphological data obtained by coronary computed tomography angiography with quantitative echocardiographic data on regional myocardial function
}

\author{
Piotr Lipiec ${ }^{1}$, Paulina Wejner-Mik ${ }^{2}$, Katarzyna Wdowiak-Okrojek ${ }^{1}$, \\ Ewa Szymczyk², Adam Skurski ${ }^{3}$, Andrzej Napieralski³ ${ }^{3}$ Marek Kamiński³, \\ Konrad Szymczyk ${ }^{4}$, Jarosław D. Kasprzak ${ }^{2}$ \\ ${ }^{1}$ Department of Rapid Cardiac Diagnostics, Medical University of Lodz, Lodz, Poland \\ ${ }^{2}$ Department of Cardiology, Medical University of Lodz, Lodz, Poland \\ ${ }^{3}$ Department of Microelectronics and Computer Science, Lodz University of Technology, Lodz, Poland \\ ${ }^{4}$ Department of Radiology, Medical University of Lodz, Lodz, Poland
}

\begin{abstract}
Background: Three-dimensional (3D) fusion of morphological data obtained by coronary computed tomography angiography (CCTA) with functional data from resting and stress echocardiography could potentially provide additional information compared to examination results analyzed separately and increase the diagnostic and prognostic value of non-invasive imaging in patients with suspected coronary artery disease (CAD). Using vendor-independent software developed in our institution, we aimed to assess the feasibility and reproducibility of $3 D$ fusion of morphological CCTA data with echocardiographic data regarding regional myocardial function.
\end{abstract}

Methods: Thirty patients with suspected CAD underwent CCTA and resting transthoracic echocardiography. From CCTA we obtained $3 D$ reconstructions of coronary arteries and left ventricle (LV). Offline speckle-tracking analysis of the echocardiographic images provided parametric maps depicting myocardial longitudinal strain in 17 segments of the LV. Using our software, 3 independent investigators fused echocardiographic maps with CCTA reconstructions in all patients. Based on the obtained fused models, each segment of the LV was assigned to one of the major coronary artery branches.

Results: Mean time necessary for data fusion was $65 \pm 7 \mathrm{~s}$. Complete agreement between independent investigators in assignment of LV segments to coronary branches was obtained in 94\% of the segments. The average coefficient of agreement (kappa) between the investigators was 0.950 and the intra-class correlation coefficient was 0.9329 (95\% CI 0.9227-0.9420).

Conclusions: Three-dimensional fusion of morphological CCTA data with quantitative echocardiographic data on regional myocardial function is feasible and allows highly reproducible assignment of myocardial segments to coronary artery branches. (Cardiol J 2016; 23, 3: 264-269)

Key words: coronary artery disease, hybrid imaging, myocardial function, echocardiography, coronary computed tomography angiography

Address for correspondence: Piotr Lipiec, MD, PhD, Department of Cardiology, Bieganski Hospital, Medical University of Lodz, ul. Kniaziewicza 1/5, 91-347 Łódź, Poland, tel/fax: +48 42 6539909, e-mail: lipiec@ptkardio.pl 


\section{Introduction}

Coronary angiography remains the reference technique in the diagnostics of coronary artery disease (CAD). However, it is an invasive examination associated with risk of complications. The qualification of patients to coronary angiography is based on clinical presentation and results of non-invasive examinations. Coronary computed tomography angiography (CCTA) is one of such modalities, but its specificity (64-83\%) in detecting significant coronary stenoses may be regarded as suboptimal, especially in patients with obesity, coronary calcifications, higher heart rates or irregular heart rhythms [1]. Other valuable non-invasive diagnostic tools include imaging techniques enabling assessment of resting and stress myocardial perfusion and function - stress echocardiography, singlephoton emission computed tomography (SPECT) and positron emission tomography (PET) $[1,2]$.

Recently, the fusion of SPECT and CCTA (SPECT/CCTA), as well as PET and CCTA (PET/ /CCTA), has been intensively studied, as it allows combining the morphological data (coronary angiogram) with functional data (resting and stress perfusion or metabolism), thus increasing the diagnostic and prognostic value of noninvasive imaging [3-5]. However, these techniques are associated with increased exposure to radiation [6]. In contrast, echocardiography is a relatively inexpensive and widely available technique, free from such risks, therefore the possibility of using the data from resting and stress echocardiography instead of functional data from PET or SPECT seems a promising and safer alternative in patients with suspected or known CAD.

Using software developed in our institution, we aimed to assess the feasibility and reproducibility of three-dimensional (3D) fusion of morphological CCTA data with functional echocardiographic data regarding regional myocardial function in patients with suspected CAD.

\section{Methods}

Thirty patients ( 14 men; mean age $60 \pm 7$ years, range $46-74$ years) with suspected CAD (mean Canadian Cardiovascular Society class $2.1 \pm$ \pm 0.7 ) referred for CCTA by their attending physicians were included in the study. Within 2 days of CCTA, they all underwent resting transthoracic echocardiography. The study protocol was approved by the Ethics Committee of our institution.

\section{Coronary computed tomography angiography}

A 64-slice multidetector computed tomography scanner (Toshiba Aquilion 64, Toshiba Medical Systems Corporation, Otawara, Japan), which is a $64 \times 0.5 \mathrm{~mm}$ collimation scanner with a gantry rotation speed of $400 \mathrm{~ms} /$ rotation, was used for CCTA. A non-enhanced prospectively electrocardiography-triggered scan was acquired for a coronary artery calcium score, followed by a contrast-enhanced scan during end-inspiratory 7-10 s breath-hold. Nonionic contrast material (Iomeron 400, Bracco) was injected with a flow rate of $4.5 \mathrm{~mL} / \mathrm{s}$. Start delay was determined by the bolus tracking technique using a region of interest in the ascending aorta. Scanning was performed at tube voltage and tube current depending on patient's body mass index. Patients with a pre-scan heart rate exceeding $65 \mathrm{bpm}$ were intravenously given metoprolol before the scan.

Scans were transferred to VitreaWorkstation 5.2, (Vital Images Inc.) for analysis. Threedimensionl volume-rendered reconstructions of the left ventricle (LV) and coronary arteries were generated for each patient.

\section{Echocardiography}

Three standard apical views (4-, 2- and 3-chamber) were acquired using Vivid 9 echocardiograph (GE Medical Systems Inc.). The images were transferred to EchoPac workstation (GE Medical Systems Inc.) for quantitative analysis using Automated Function Imaging package based on speckle tracking algorithm. Bull's-eye maps representing peak systolic longitudinal strain in $17 \mathrm{LV}$ segments were generated for each patient. Patients with quality of echocardiographic images precluding speckle tracking analysis of all LV segments were excluded from the study.

\section{Fusion of CCTA with echocardiographic data}

Using vendor-independent software developed in our institution, 3 independent investigators fused CCTA-based 3D reconstructions of LV and coronary arteries with echocardiographic bull's-eye maps in all patients. Time necessary for data fusion was measured in each case. Based on obtained fused models, the investigators independently assigned each segment of the LV to one of the major coronary artery branches (left anterior descending artery, diagonal branch, left circumflex artery, left marginal branch, right coronary artery). 


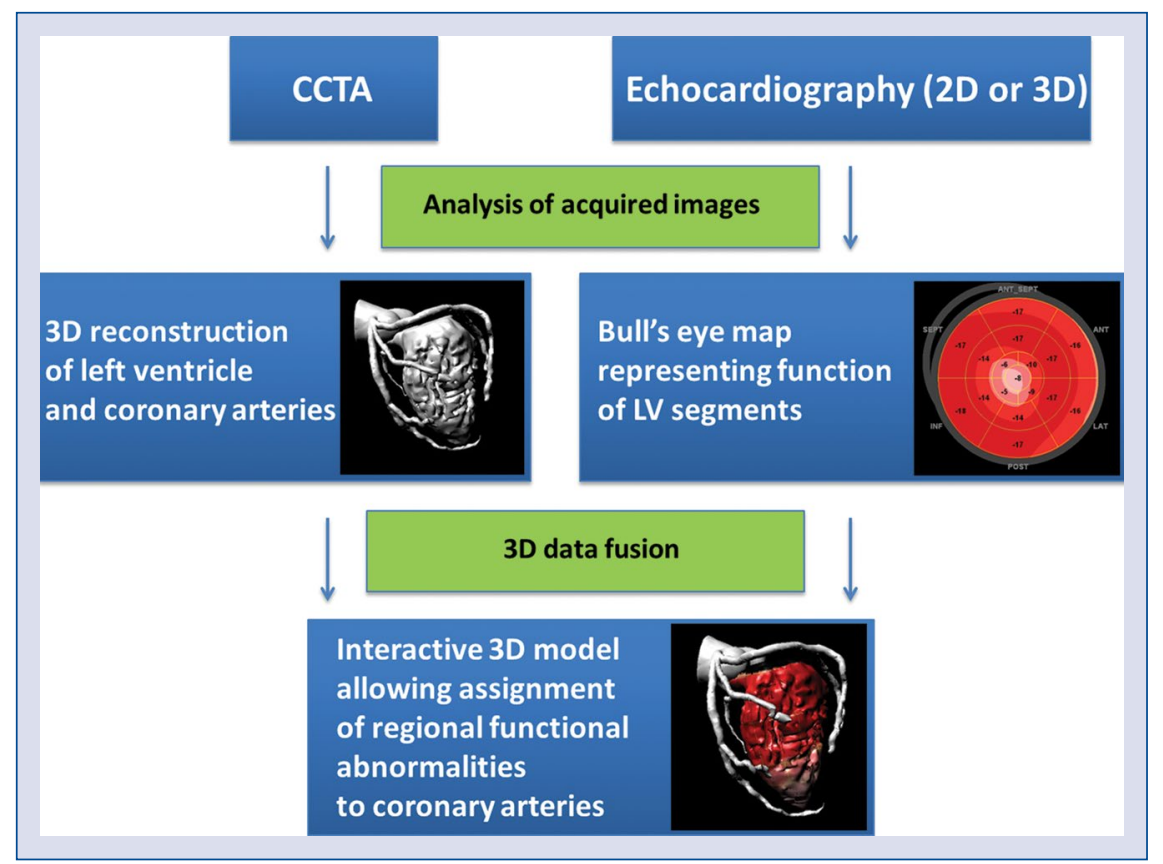

Figure 1. Three-dimensional (3D) fusion of coronary computed tomography angiography (CCTA) with echocardiographic functional data - schematism of algorithm and software developed in our institution and example of obtained 3D fused model. Full explanation in the text.

Our software, which can be deployed on any PC station without any constrains of hardware and software configuration, requires uploading three files for each patient: two STL (StereoLithography) files representing CCTA-based $3 \mathrm{D}$ reconstructions of the LV and the coronary arteries and one JPG, TIFF or PNG file containing echocardiographic bull's-eye map. It should be noted that all these file formats are standardized and supported by numerous software packages and, therefore, our software allows vendor-independent analysis.

Merging 2-dimensional (2D) bull's-eye maps with a $3 \mathrm{D}$ model requires accurate orientation, which is achieved by mapping each point of a 2D image onto sections of the 3D LV model. We use a semi-automatic solution, in which the user is asked to manually place 3 equivalent markers both on the $3 \mathrm{D}$ model of the $\mathrm{LV}$ and on the $2 \mathrm{D}$ map (1 marker at apex of the LV and 2 markers at the base of the LV: one on the border between anterior septum and the anterior wall and the second one on the border between inferior septum and the inferior wall). This provides enough information for executing a fully automated matching and texturing algorithms. Custom texturing (UV mapping technique) is used to merge the two different domain (3D and 2D) images into one interactive view. The last stage (fully automatic) of the fusion procedure is adding the $3 \mathrm{D}$ reconstruction of the coronary arteries.

The final result of data fusion is an interactive 3D model, which allows allocation of LV segments to coronary arteries and is supposed to help in recognizing whether possible coronary stenosis observed on CCTA is associated with possible abnormality in myocardial function (Fig. 1).

\section{Statistical analysis}

Continuous and categorical variables are expressed as mean \pm standard deviation and as percentages (\%), respectively. The agreement between investigators in assignment of LV segments to one of major coronary branches was assessed using inter-rater agreement statistic (kappa) and the the intra-class correlation coefficient.

\section{Results}

Three-dimensional data fusion was feasible in all cases. The mean time necessary for data fusion was $65 \pm 7 \mathrm{~s}$ (range: 51-79 s). Complete agreement between independent investigators in assignment of LV segments to one of major coronary branches was obtained in 481 (94\%) segments (Table 1). The average coefficient of agreement (kappa) between 
Table 1. Agreement between investigators in assignment of 510 left ventricular segments to major coronary artery branches in 30 patients.

\begin{tabular}{lcc}
\hline Investigator & Complete agreement & Coefficient of agreement (kappa) \\
\hline 1 vs. 2 & $496(97 \%)$ segments & $0.965(95 \% \mathrm{Cl}: 0.947-0.983)$ \\
1 vs. 3 & $490(96 \%)$ segments & $0.950(95 \% \mathrm{Cl}: 0.928-0.971)$ \\
2 vs. 3 & $484(95 \%)$ segments & $0.935(95 \% \mathrm{Cl}: 0.910-0.959)$ \\
\hline
\end{tabular}

$\mathrm{Cl}$ - confidence interval

the investigators was 0.950 and the intra-class correlation coefficient was 0.9329 (95\% confidence interval 0.9227-0.9420).

Left ventricular segments most frequently assigned to different major coronary artery branches by independent investigators were: apical lateral (discrepancies in 7 cases), apical inferior (discrepancies in 6 cases), apical septal (discrepancies in 6 cases), mid inferolateral (discrepancies in 4 cases) and mid anterolateral (discrepancies in 2 cases).

\section{Discussion}

To the best of our knowledge, this is the first study documenting feasibility and reproducibility of vendor-independent 3D fusion of morphological CCTA data with functional echocardiographic data in patients with suspected CAD. In the literature, we found only 1 case report describing feasibility of vendor-specific fusion of data from 3D speckle tracking echocardiography with CCTA images [7].

We used echocardiographic bull's-eye maps representing peak systolic longitudinal strain due to the fact that it is a well validated parameter of myocardial systolic function and it can be also used for quantitative analysis of a stress test, as demonstrated by our group and other investigators $[8,9]$. However, it is also possible to fuse maps representing other echocardiographic parameters of regional function, such as strain rate.

Based on encouraging results available in the literature, showing incremental clinical value of hybrid PET/CCTA and SPECT/CCTA imaging in patients with suspected CAD, one can assume that fusion of morphological data obtained by CCTA with functional data from resting and stress echocardiography could potentially provide additional information compared to examination results analyzed separately, and could increase the diagnostic and prognostic value of non-invasive imaging in this patient population (Fig. 2). Nevertheless, this assumption and the choice of most suitable echocardiographic parameters to fuse with CCTA data require validation in further clinical studies.

In our study, we obtained functional data from 2D echocardiography. Functional maps can also be created from 3D echocardiographic datasets, although 3D echocardiography remains less widely available and inferior in temporal resolution to $2 \mathrm{D}$ techniques, which is an important limitation in functional analysis. Furthermore, functional parameters derived from quantitative analysis of $2 \mathrm{D}$ echocardiographic images are still more validated than those obtained by $3 \mathrm{D}$ echocardiography $[10,11]$.

\section{Limitations of the study}

This is a single-center study on a relatively small group of patients. However, such preliminary feasibility and reproducibility data are required before this technique can be evaluated in larger clinical studies. Another limitation of this study is the use of only 1 computed tomography scanner and 1 echocardiograph, instead of equipment provided by various vendors. Since our software allows using file formats, which are standardized and supported by numerous software packages (STL, JPG, TIFF or PNG), we believe that it can also be used for fusion of data acquired with other computed tomography scanners and echocardiographs. Nevertheless, this assumption requires validation.

It should also be underlined that our findings regarding feasibility and reproducibility of CCTA-echocardiography fusion are based on analysis of only resting echocardiographic images. However, we believe that these results can be extrapolated to fusion of CCTA data with stress echocardiography. Our software tool uses echocardiographic bull's-eye maps, which can be obtained with various analysis packages (in our case EchoPac, GE Medical Systems) at low and high heart rates — the feasibility of obtaining strain data and bull's-eye maps during stress echocardiography, as well as their diagnostic value, have been presented in numerous papers, including the one from our group [8,9]. 


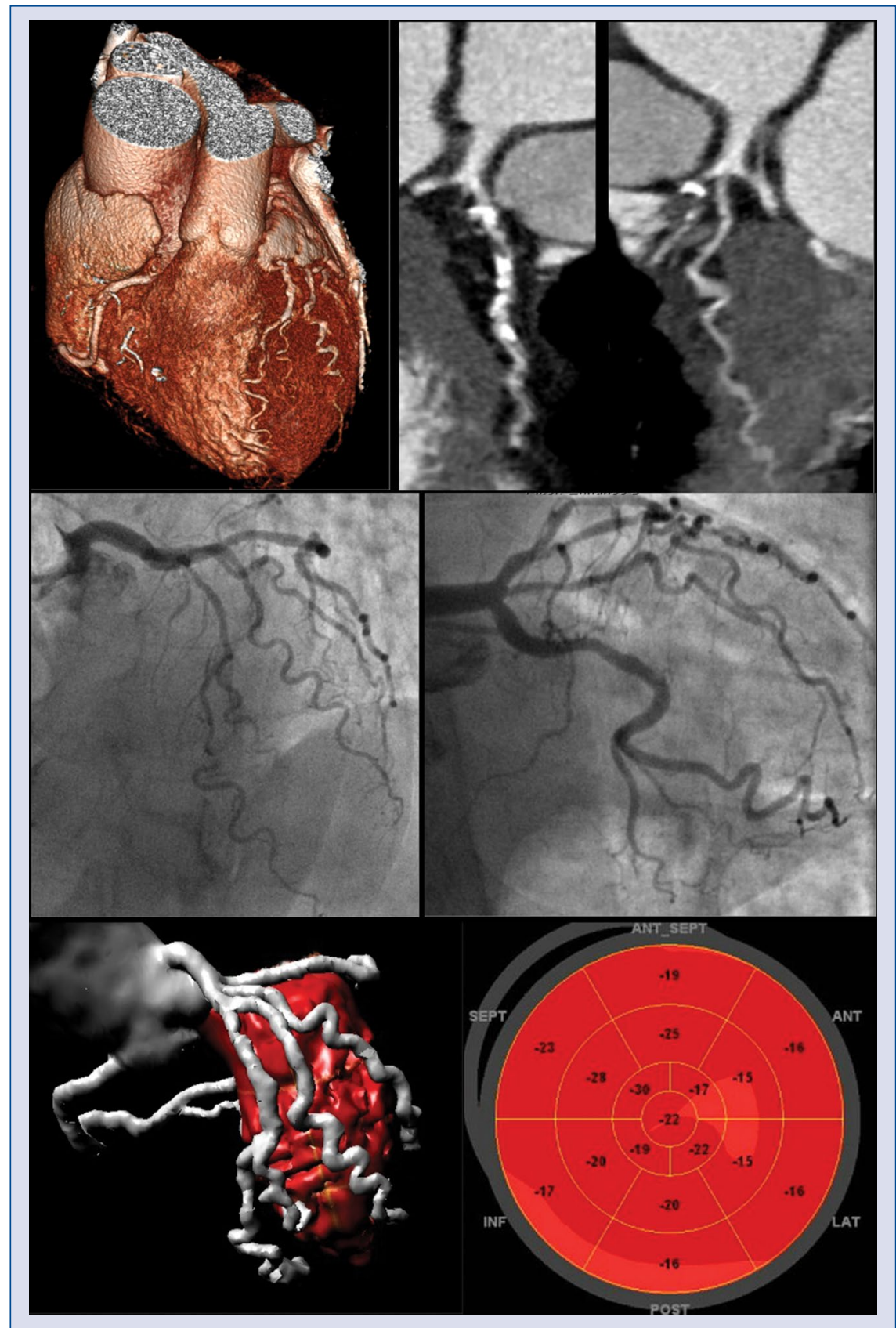

Figure 2. Diagnostic evaluation of a 66-year-old female patient with symptoms of stable coronary artery disease. Upper panel: Coronary computed tomography angiography (CCTA) revealed lesions of proximal left anterior descending coronary artery and intermediate coronary artery, as well as suspected lesion in proximal large first diagonal branch; Middle panel: Invasive coronary angiography confirmed stenoses of these three coronary branches; Lower panel: Quantitative analysis of stress echocardiography images revealed lower longitudinal strain within anterior and lateral walls with the area of lowest strain within the middle segments of these walls. Three-dimensional fusion of CCTA with stress echocardiographic functional data indicated that this area can be allocated to the large first diagonal branch.

\section{Conclusions}

Three-dimensional fusion of morphological CCTA data with quantitative echocardiographic data on regional myocardial function is feasible, rapid and allows highly reproducible assignment of myocardial segments to coronary artery branches. Further studies in patients with suspected and known CAD seem warranted and are needed to provide data on the diagnostic and prognostic value of this technique. 
Conflict of interest: Piotr Lipiec and Jarosław D. Kasprzak have received speaker fees from GE Medical Systems Inc.

\section{References}

1. Montalescot G, Sechtem U, Achenbach S et al. 2013 ESC guidelines on the management of stable coronary artery disease: The Task Force on the management of stable coronary artery disease of the European Society of Cardiology. Eur Heart J, 2013; 34: 2949-3003. doi: 10.1093/eurhearti/eht296.

2. Wolk MJ, Bailey SR, Doherty JU et al. ACCF/AHA/ASE/ASNC/ HFSA/HRS/SCAI/SCCT/SCMR/STS 2013 multimodality appropriate use criteria for the detection and risk assessment of stable ischemic heart disease: A report of the ACCF Appropriate Use Criteria Task Force, AHA/ASE/ASNC/HFSA/HRS/SCAI/SCCT/ SCMR/STS. J Am Coll Cardiol, 2014; 63: 380-406. doi: 10.1016/j. jacc.2013.11.009.

3. Dong W, Wang Q, Gu S, Su H, Jiao J, Fu Y. Cardiac hybrid SPECT/ /CTA imaging to detect "functionally relevant coronary artery lesion": A potential gatekeeper for coronary revascularization? Ann Nucl Med, 2014; 28: 88-93. doi: 10.1007/s12149-013-0790-9.

4. Schaap J, de Groot JA, Nieman K et al. Added value of hybrid myocardial perfusion SPECT and CT coronary angiography in the diagnosis of coronary artery disease. Eur Heart J Cardiovasc Imag, 2014; 15: 1281-1288. doi: 10.1093/ehjci/jeu135.

5. Flotats A, Knuuti J, Gutberlet $M$ et al. Hybrid cardiac imaging: SPECT/CT and PET/CT. A joint position statement by the European Association of Nuclear Medicine (EANM), the European Society of Cardiac Radiology (ESCR) and the European Council of Nuclear Cardiology (ECNC). Eur J Nucl Med Mol Imag, 2011; 38: 201-212. doi: 10.1007/s00259-010-1586-y.

6. Gerber TC, Carr JJ, Arai AE et al. Ionizing radiation in cardiac imaging: a science advisory from the American Heart Association Committee on Cardiac Imaging of the Council on Clinical Cardiology and Committee on Cardiovascular Imaging and Intervention of the Council on Cardiovascular Radiology and Intervention. Circulation, 2009; 119: 1056-1065. doi: 10.1161/ CIRCULATIONAHA.108.191650.

7. Casas Rojo E, Fernández-Golfín C, Zamorano J. Hybrid imaging with coronary tomography and 3D speckle-tracking stress echocardiography fusion. Eur Heart J Cardiovasc Imag, 2014; 15: 555. doi: 10.1093/ehjci/jet236.

8. Wierzbowska-Drabik K, Hamala P, Roszczyk N et al. Feasibility and correlation of standard 2D speckle tracking echocardiography and automated function imaging derived parameters of left ventricular function during dobutamine stress test. Int J Cardiovasc Imag, 2014; 30: 729-737. doi: 10.1007/s10554-014-0386-z.

9. Mitro P, Murin P, Valocik G, Simurda M. Regional myocardial contractile reserve assessed by strain echocardiography and the response to cardiac resynchronization therapy. Cardiol J, 2014; 21: 524-531. doi: 10.5603/CJ.a2014.0003.

10. Muraru D, Cucchini U, Mihăilă S et al. Left ventricular myocardial strain by three-dimensional speckle-tracking echocardiography in healthy subjects: reference values and analysis of their physiologic and technical determinants. J Am Soc Echocardiogr, 2014; 27: 858-871. doi: 10.1016/j.echo.2014.05.010.

11. Lang RM, Badano LP, Mor-Avi V et al. Recommendations for cardiac chamber quantification by echocardiography in adults: An update from the American Society of Echocardiography and the European Association of Cardiovascular Imaging. Eur Heart J Cardiovasc Imag, 2015; 16: 233-271. doi: 10.1093/ehjci/jev014. 